\title{
ENJEUX ET POLITIQUES FONCIĖRES EN FRANCE ET AU BRÉSIL: REGARDS CROISÉS SUR DEUX HISTOIRES CONTRASTÉES
}

\author{
LAND OWNERSHIP POLICY CHALLENGES \\ IN FRANCE AND BRAZIL: \\ A LOOK AT TWO CONTRASTING HISTORIES
}

\author{
Yannick Sencébé* \\ Ademir Antonio Cazella**
}

\section{Introduction}

Le Brésil, géant vert dont la croissance repose sur l'exportation de produits agricoles reste aussi l'un des pays les plus inégalitaires en termes de structure foncière. En effet, moins de 1\% des exploitations (46.911) détient 44,4\% de la surface totale des unités agricoles. Tandis que les 4.448 .648 exploitations de moins de 100 ha (86\%) possèdent à peine $21,4 \%$ de cette surface (IBGE, 2006).

Son modèle de développement repose en grande partie sur la dualité de son agriculture. La grande agriculture d'entreprise produit pour l'exportation et génère ainsi des devises pendant que l'agriculture dite familiale nourrit la population brésilienne, comme le rappelle le Ministère du Développement Agraire (MDA) ${ }^{1}$. Ce ministère, créé en 1999, marque la reconnaissance officielle de l'agriculture "familiale" avec pour mission la mise en place de politiques de développement rural. Il cohabite cependant avec le Ministère de l'Agriculture, dédié au secteur de "l'agronegócio" (agrobusiness). Cette partition ministérielle peut surprendre, mais elle mériterait d'être mise en regard avec les ambivalences qui ont traversé, en France, la politique foncière et agricole au sein du même ministère (installation/ agrandissement; nourrir la France / exporter; productivisme / multifonctionnalité...). La France

\footnotetext{
* É professora de sociologia no Instituto Nacional Superior das Ciências Agronômicas, da Alimentação e do Meio Ambiente (Agrosup) e no Centro de Economia e de Sociologia Aplicadas à Agricultura e aos Espaços Rurais (Cesaer /Paris/ FR). yannick.sencebe@dijon.infra.fr.

** É professor e atual coordenador do Programa de Pós-graduação em Agroecossistemas da Universidade Federal de Santa Catarina (ppgagro/UFSC/Florianópolis/SC/BR).ademir.cazella@ufsc.br

1. Selon les chiffres du MDA, plus des 2/3 de l'alimentation consommée dans le pays est produite par l'agriculture familiale.
} 
peut être considérée, elle aussi, comme un grand pays agricole, et sans doute l'un des derniers bastions paysans d'Europe, sa modernisation agricole ayant été très tardive. De ce fait, l'agriculture continue à y occuper une place dans la vie politique et dans l'imaginaire national plus que proportionnelle à ses effectifs (HERVIEU; VIARD, 2001).

Nous proposons ainsi une mise en regard de ces deux pays, où l'agriculture tient une place particulière, avec cependant des trajectoires et des enjeux contrastés. Plus précisément, nous centrons ce regard sur la question foncière qui a fortement marqué leurs histoires respectives et qui prend, avec le contexte de crise alimentaire et environnementale, un relief particulier. Au Brésil, la réforme agraire, toujours à l'état de débat et de lutte socio-politique, renvoie à la question de la souveraineté alimentaire interne, notamment pour les très nombreux petits paysans, mais aussi à des enjeux de modèle de développement. En effet, la grande agriculture capitaliste dédiée à l'exportation monopolise la plus grande partie des terres agricoles dans les régions les plus peuplées et développées. Ce quasi-monopole reporte sur les fronts pionniers de l'Amazonie ou encore dans le Nord-Est une bonne partie des paysans sans terre qui participent ainsi à la déforestation (Amazonie) et survivent dans des conditions de vie très précaires (le Nord-Est étant une région très pauvre et touchée par la sécheresse).
En France, dans une moindre mesure, s'observe un partage inégalitaire entre l'agriculture spécialisée et dédiée à l'exportation (céréaliculture principalement) et l'agriculture que l'on pourrait dénommer "territoriale”, fondée sur des marchés de proximité et des liens forts avec le territoire: services à la population, activités agro-touristiques et produits de terroirs (SENCÉBÉ, 2011). Celle-ci bénéficie de la remise en question $\mathrm{du}$ modèle productiviste (crises sanitaires, coûts sociaux et environnementaux) et de la demande croissante, plutôt urbaine, en produits de proximité et de qualité. Mais l'accès à la terre reste très difficile pour ce type d'agriculture et, plus largement, la concurrence foncière s'exacerbe entre agriculteurs, mais aussi entre usages de la terre.

L'enjeu foncier de part et d'autre de l'Atlantique est au cœur non seulement de la question socio-politique d'un partage plus équitable des terres et de la régulation de l'accès au foncier, mais aussi au centre de la redéfinition des modèles agricoles susceptibles de répondre en les articulant aux enjeux alimentaires, sociaux et environnementaux actuels.

Cette analyse est issue d'un travail exploratoire mené par ses auteurs français et brésilien dans la perspective d'une recherche comparée sur les questions foncières au sein de ces deux pays. Nos données sont issues de travaux de terrain menés en parallèle et en commun ${ }^{2}$ et de

2. L'auteure française a travaillé sur le contrôle et le partage du foncier dans le cadre d'un programme de recherche PSDR INRA Bourgogne (TERAGRI, 2009-2011). L'auteur brésilien travaille depuis des années sur l'agriculture familiale, la multifonctionnalité, les politiques de crédit foncier au Brésil. Ensemble, ils ont encadré différents travaux universitaires sur le foncier en France et au Brésil, ont mené une analyse des politiques foncières en France en en tirant les enseignements pour le Brésil. Enfın, plus récemment un terrain commun a été exploré dans l'État du Piauí, concernant l'installation collective de paysans sans terre avec l'appui du crédit foncier, au-delà de l'organisation d'une mission scientifique et technique en France de dirigeant du MDA et de syndicalistes brésiliens, qui traitent de la question de l'accès à la terre. 
la lecture croisée de sources statistiques et bibliographiques concernant l'histoire et les politiques foncières et agricoles des deux pays. Nous commencerons donc par brosser le tableau de départ: celui des enjeux fonciers au regard de la situation agricole, française et brésilienne, actuelle. Nous procéderons ensuite à une remontée dans leur histoire agraire et foncière pour comprendre les objectifs et les rapports de force à l'origine des politiques foncières et qui peuvent expliquer la situation actuelle de l'agriculture. Enfin, nous tirerons les enseignements de ces deux histoires en portant un regard croisé sur les limites et les points forts des politiques foncières françaises et brésiliennes.

\section{Les données de l'enjeu foncier de part et d'autre de I'Atlantique}

La démographie agricole suit des trajectoires inversées dans les deux pays. Alors qu'en France la fonte des effectifs agricoles est un phénomène ancien et tendanciel, le Brésil voit augmenter le nombre de ses exploitations. Le dernier recensement agricole en France 2010 pointe ainsi une diminution de $26 \%$ du nombre d'exploitations en dix ans (AGRESTE PRIMEUR, 2011). Des 2,3 millions d'exploitations qui occupaient le territoire en 1945, il n'en reste plus guère que 500.000, dont la surface moyenne ne cesse d'augmenter (55 ha en moyenne en 2010). Au Brésil, au contraire, le nombre d'exploitations reste très important et l'analyse des recensements agricoles sur le plus long terme dévoile une tendance à la hausse depuis les années 1970 (Figure 1) (3) $^{3}$

Figure 1 - Nombre d'exploitations agricoles au Brésil

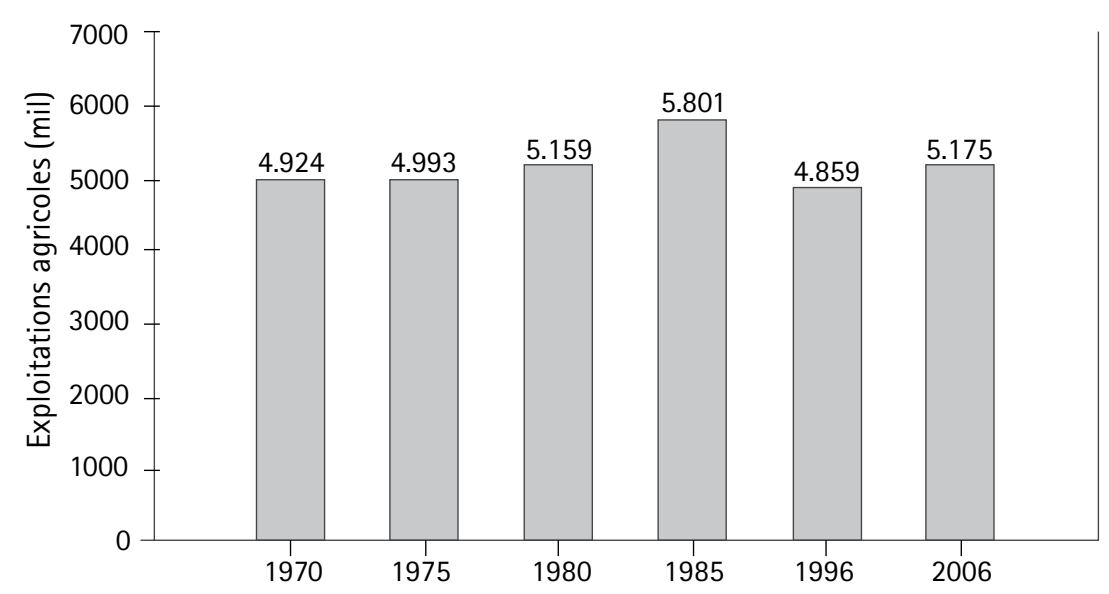

Sources: NUNES (2008), modifié par les auteurs.

3. En 1996, la baisse du nombre d'établissements pourrait être due à certains changements de méthode de collecte des données, notamment le fait qu'une partie des agriculteurs non propriétaires n'auraient pas été recensés. 
Ces trajectoires presque inversées convergent cependant sur une même tendance: la dualisation de l'agriculture. L'usage que nous faisons de la notion de dualisation intégre deux dimensions du procesus qui sont corrélés. La première a trait à l'accès à la terre profondément inégal entre différents types d'agriculteurs. L'opposition est particulièrement claire de ce point de vue entre les exploitations agricoles familiales et les exploitations patronales (dans lequel prédomine le travail salarié), mais il existe également une dualité interne entre les exploitations familiales. La seconde dimension se réfere l'inégalité d'accès aux politiques publiques de dévelppement rural et agricole, avec une tendance très claire de ces politiques à privilégier les exploitations considérées comme productives (qu'elles soient familiales ou non) au regard des indices de productivité technique. Au Brésil la dualité de l'agriculture s'exprime par "le bas": 84\% des 5 millions d'exploitations du pays sont considérés comme "familiales" (4.367.902) La distinction entre les unités agricoles familiales ou non représente une innovation du dernier recensement et traduit bien, dans la statistique officielle, la reconnaissance de ce type d'agriculture ${ }^{5}$. L'agriculture familiale représente ainsi 74\% des actifs occupés en milieu rural (12,5 millions de personnes). Cependant, si tous les paysans pauvres relèvent de l'agriculture familiale, tous les agriculteurs familiaux ne sont pas pauvres. On pourrait parler d'une certaine dualité interne à l'agriculture familiale, ce qui complique d'ailleurs la tâche du MDA. Une part de cette agriculture, notamment au sud du pays, est composée d'agriculteurs intégrés à l'agro-industrie, très performants et dominant, en partie sinon en totalité, certaines productions (poulet et porc, lait). L'autre partie vit dans la misère, de l'autoconsommation de manioc et de haricots et, depuis peu, grâce aux bourses familiales (mesure mise en place par Lula).

En France, la dualité de l'agriculture s'exprime plutôt par le "haut", l'immense majorité des "petites" exploitations d'aprèsguerre n'ayant cessé de disparaitre au profit de l'agrandissement des "moyennes" et de la constitution plus récente de grandes entreprises agricoles (Figure 2).

4. Le dernier recensement agricole réalisé au Brésil date de 2006 et le pays ne dispose d'aucune source statistique plus récente qui se réfère à la notion d'établissement agricole" utilisée dans le recensement. Notre ambition étant de réaliser une analyse comparative à partir d'une même base conceptuelle, nous avons choisi de n'utiliser que les données du recensement malgré leur relative ancienneté.

5. Selon la loi de Juillet 2006, l'agriculture familiale doit respecter, à la fois, les critères suivants: 1) Ne pas posséder, sous quelque statut que ce soit, une surface supérieure à 4 modules fiscaux (leur taille varie selon les conditions technico-productives des différentes microrégions du pays); 2) Utiliser de façon prédominante la main d'œuvre familiale dans les activités économiques de l'exploitation; 3) Tirer le revenu familial principalement des activités économiques de l'exploitation; 4) Diriger l'exploitation avec la famille. 
Figure 2 - Le comportement des petites, moyennes et grands exploitations agricoles entre les recensements agricoles de 2000 et 2010 en France.

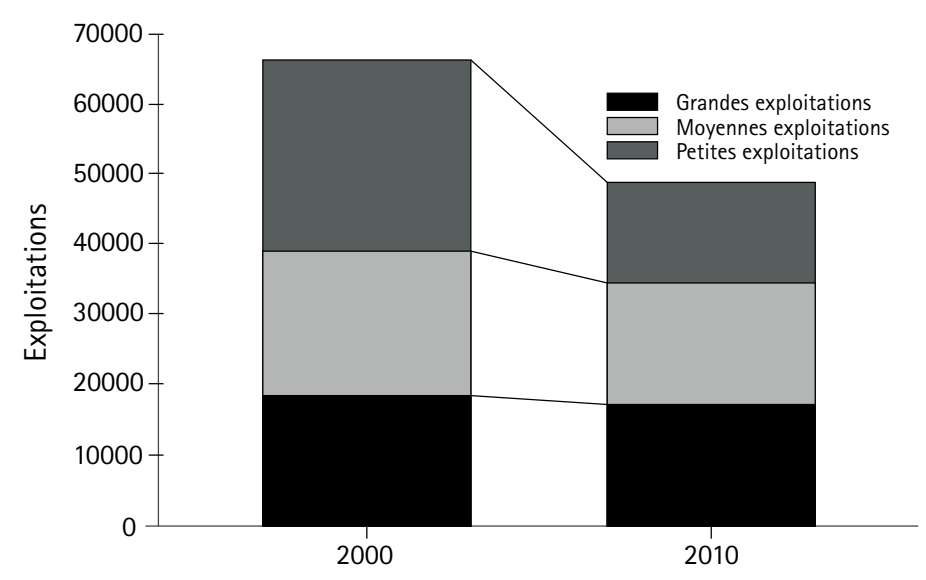

Sources: Agreste - Recensements agricoles

Cette partition triptyque, innovation $d u$ dernier recensement agricole en France, redéfinit la valeur accordée à la taille des exploitations, auparavant fondée sur les résultats économiques (à travers la Marge Brute Standard), et dorénavant basée sur le potentiel de production des exploitations exprimé en Production Brute Standard ${ }^{6}$. Il réintroduit dans la classification les "petites” exploitations auparavant considérées comme "non professionnelles" et traitées à part quand elles étaient prises en compte ${ }^{7}$. Représentant encore 36\% des exploitations et un quart des actifs agricoles, son rôle social, humain et territorial est dorénavant souligné dans les publications officielles de la statistique agricole française (AGRESTE PRIMEUR, 2011).

Ainsi est-il assez révélateur que de part et d'autre, la petite agriculture soit nouvellement prise en compte dans les statistiques officielles, soit parce qu'elle s'impose au Brésil comme secteur social et économique incontournable au prix de longues luttes sociales et politiques (SCHNEIDER; CAZELLA; MATTEI, 2004; CAZELLA; BONNAL; MALUF, 2009; DELGAD0, 2010), soit parce qu'en s'effaçant comme secteur économique, d'autres fonctions lui sont reconnues ou conférées comme en France.

6. D'après Agreste: la Production Brute Standard (PBS) décrit un potentiel de production des exploitations (calculé sur plusieurs années, chaque type de production étant référé à un coefficient qui définit la valorisation du rendement sur le marché) et permet de les classer selon leur dimension économique. Sont ainsi considérées comme moyennes les exploitations dont la PBS est supérieure ou égale à 25000 euros, et comme grandes, les exploitations dont la PBS est supérieure ou égale à 100000 euros. Les petites n'apparaissant ainsi que comme solde en dessous de 25000 euros.

7. Les moyennes et grandes exploitations sont néanmoins regroupées pour constituer le nouveau champ du Réseau d'Information Comptable Agricole (RICA) instrument européen qui permet d'évaluer le revenu des exploitations et l'impact de la politique agricole communautaire. Les petites exploitations en sont donc exclues. 
Ces trajectoires historiques différentes amènent finalement à des ratios très inégalitaires des deux côtés de l'Atlantique. Ainsi en France, les grandes exploitations (au nombre de 161.500) assurent désormais plus de $80 \%$ du potentiel de production (contre $73 \%$ en 2000), le reste relevant pour 17\% des moyennes (au nombre de 150.500) et pour 3\% des 178.000 petites exploitations (AGRESTE PRIMEUR, 2011). Au Brésil, à peine 8,2\% des établissements (423.689 exploitations) assurent près de $85 \%$ de la Valeur Brute de la Production (VBP) agricole ${ }^{8}$. La frange intermédiaire, représentant 18,8\% des établissements (975.974), génère 11,1\% de la VBP et les 73\% restant (3.775.826 exploitations) se partagent à peine $4 \%$ de la VBP (ALVES; ROCHA, 2010). Notons que les valeurs calculées ici sont monétaires ce qui explique que l'agriculture familiale, principalement représentée dans le 3ème groupe, soit en même temps responsable de la production des 2/3 de l'alimentation des brésiliens, données élaborées par le MDA en quantité produite: or la valeur monétaire de la production destinée au marché intérieur est bien moins élevée que celle des produits exportés (concentrés dans le $1^{\mathrm{er}}$ groupe).

Pour autant, la situation des petites exploitations est bien différente, tout comme les enjeux soulevés par l'inégale répartition $\mathrm{du}$ foncier. Au Brésil, la question agraire et foncière pourrait se résumer ainsi: de très petites exploitations en situation de pauvreté et d'insécurité foncière voient leur nombre augmenter plus vite que la part du "gâteau" foncier et économique qui leur est réservé. En France, à l'inverse, des exploitations toujours moins nombreuses sur des surfaces toujours plus grandes, tournées vers des marchés lointains, accaparent l'essentiel des terres libérées dans un contexte de forte concurrence foncière, ce qui limité non seulement le renouvellement des exploitations mais aussi l'installation de nouvelles formes d'exploitations répondant à une demande pourtant croissante (agriculture biologique, de proximité).

Au Brésil le recensement de 2006 a révélé la persistance de graves problèmes d'accès à la terre. Parmi les 3.946.276 d'agriculteurs propriétaires, 46,6\% (1.840.734) ont une surface inférieure à 2 ha et, pour une partie d'entre eux, sont forcés de vendre leur force de travail de façon précaire et saisonnière pour survivre (CAZELLA; BÚRIGO, 2011). On ne compte pas moins de 1.040.022 agriculteurs (un peu plus d'1/5 du total) qui ne sont pas propriétaires de leurs exploitations. Qu'ils soient "occupants", fermiers ou métayers, leur situation foncière est précaire. Dans le premier cas, les familles utilisent des terres sans titre de propriété, parfois depuis des générations. Quant aux autres, dans la majorité des cas, les contrats de fermage et de métayage concernent de petites surfaces et ont un statut précaire, leur durée étant d'à peine une saison alors que la législation exige une durée minimale de 3 ans. En effet, mal informés, beaucoup de propriétaires fonciers, craignant d'être pénalisés par la loi d'usucapion ${ }^{10}$, limitent

8. La VBP correspond au volume de la production totale (incluant l'autoconsommation) multiplié par sa valeur sur le marché. Elle est assez proche de la Production Brute Standard.

9. Certains syndicats, la Confédération Paysanne notamment, les désignent sous le terme de squatters ruraux. 10. La législation brésilienne prévoit que l'occupation d'une aire inférieure à 50 ha en zone rurale durant une période de 5 ans ininterrompus et sans contestation d'un tiers donne le droit à l'occupant d'en requérir le titre de propriété. 
à une saison les contrats de fermage qu'ils accordent sur leurs domaines. Les familles, dès lors contraintes à rechercher sans cesse de nouvelles terres pour s'établir, sont confrontées une forte insécurité notamment alimentaire. Leur présence éphémère sur les exploitations louées compromet la production traditionnelle pour l'autoconsommation qui remplit un rôle important de sécurité alimentaire au sein de l'agriculture familiale.

La situation française est, de ce point de vue, bien différente. Bien que la pauvreté ne soit pas absente des campagnes et de l'agriculture (IGAS, 2009), la petite agriculture est composée avant tout de doubles actifs et d'agriculteurs retraités. La dimension géographique tient là aussi un rôle important puisqu'une partie essentielle des personnes concernées se situent dans les départements de montagne et les zones défavorisées (AGRESTE PRIMEUR, 2011).

Le problème majeur en France est la très forte concurrence foncière qui s'observe autant entre agriculteurs qu'entre usages de l'espace. La concurrence entre agriculteurs est d'autant plus forte que, du fait de la PAC, leurs revenus sont en grande partie liés à la surface qu'ils occupent. Dès lors, la moindre parcelle qui se libère attire de nombreux candidats et ceux qui sont déjà les mieux lotis du point de vue foncier sont souvent ceux qui l'emportent. En effet, les agriculteurs partant à la retraite sans successeur ont tendance à privilégier les mieux installés, d'une part en cas de vente, pour valoriser au mieux leur patrimoine foncier qui assurera, plus que leur maigre retraite, leur subsistance, d'autre part, en cas de fermage, pour s'assurer que le patrimoine familial constitué sur plusieurs générations sera "bien mis en valeur" par le voisin que l'on connait et reconnait comme profes- sionnel (BOINON, 2011). Cette situation désavantage particulièrement les candidats cherchant à s'installer "hors du cadre familial" et notamment ceux qui ne sont pas issus du milieu agricole (GILLET, 1999). Pour autant, les "hors cadres familiaux" représentent 30\% des installations de moins de 40 ans, chiffre qui ne cesse d'augmenter, et cet apport extérieur apparait essentiel pour assurer le renouvellement des générations d'agriculteurs (BERNIER, 2005). Dans tous les cas, mais davantage encore pour les hors cadres familiaux, le frein principal à l'installation est l'accès au foncier, sachant que seul 50\% des terres libérées chaque année servent à l'installation, 40\% alimentent l'agrandissement des structures existantes et 10\% partent à l'urbanisation (BERNIER, 2005).

Cette guerre pour la terre n'est pas pour rien dans le retrait relatif de l'agriculture face à l'urbanisation. En s'agrandissant du point de vue foncier, les agriculteurs diminuent leur surface sociale et leur capacité d'expression et de résistance collective. La propriété agricole reste encore dans des mains agricoles (1/3 aux agriculteurs, $1 / 3$ à leur famille et $1 / 3$ hors agriculture et hors famille agricole, selon la dernière enquête structure qui date malheureusement de 1992). Mais l'attachement à la terre devient fragile. En effet, le directeur d'une Société d'Aménagement Foncier et d'Etablissement Rural (SAFER) nous confrait ainsi lor d'un entretien : "Chacun a l'espoir que le loto tombe: que sa propriété ou un bout soit classé en constructible. On reste attaché mais jusqu'à un certain prix !". Ce sont ainsi 279000 ha de terres qui ont quitté leur vocation agricole entre 2006 et 2009 selon l'enquête Terutis (AGRESTE PRIMEUR, 2010).

L'artificialisation s'accélère, principalement à travers le développement résiden- 
tiel (AGRESTE PRIMEUR, 2009) et concerne l'équivalent d'un département (61000 ha) tous les 7 ans, prioritairement au dépend de l'agriculture. Ce sont aussi les meilleures terres du point de vue agronomique qui sont concernées (CGDD, 2011) du fait d'une certaine ironie de l'histoire: les villes, à travers leur fonction à la fois commerciale et stratégique (de défense), s'étant implantées au centre des bassins de production agricoles, grignotent aujourd'hui les plaines et les vallées qui les ont jusque-là nourries. Du côté des acteurs urbains et périurbains la situation est complexe. Une prise de conscience se fait jour quant à l'importance du maintien d'une agriculture périurbaine: circuits courts, cadre de vie, services à la population en sont les maitres mots. Mais cette sensibilité portée par les élus urbains et une partie des habitants recouvre un certain nombre d'ambigüités et de malentendus. La recherche du confort spatial et d'un environnement rural, par les nombreux habitants quittant les villes, alimente la consommation d'espace et la flambée des prix de plus en plus loin dans les couronnes périurbaines. La défense d'une agriculture paysagère et de service par les élus soucieux du cadre de vie de leurs habitants ne rencontre pas toujours l'assentiment de la profession qui s'estime non reconnue dans sa diversité (céréales, élevages) et dans son rôle nourricier. Les élus, qui ont acquis des compétences urbanistiques importantes à l'échelle des communes, n'ont pas les mêmes objectifs. Si les élus des pôles urbains ont intérêt à ré-densifier les villes et à limiter l'étalement résidentiel, les élus $d u$ périurbain et du rural sont, pour un grand nombre d'entre eux, engagés dans la course à l'attractivité résidentielle, qui alimente au final le grignotage des terres.

\section{Histoires agraires et politiques foncières}

Face à ces enjeux, de quels outils et politiques les deux pays se sont-ils dotés? La situation décrite plus haut étant contemporaine, il nous faut aussi considérer la question dans son sens inverse: dans quelle mesure les politiques agraires ont-elles contribué à l'émergence des problèmes fonciers évoqués? Il s'agit ici de revenir sur l'histoire des politiques agraires avec une attention particulière à la dimension foncière afın de comprendre les choix effectués en termes de modèle agricole, de répartition et de gestion $\mathrm{du}$ foncier.

\subsection{En France: grandeur et décadence du gouvernement professionnel du foncier agricole}

L'établissement d'un véritable "gouvernement professionnel" $\mathrm{du}$ foncier agricole fait référence à la période d'après guerre qui a vu la "profession agricole" - en même temps qu'elle se structurait solidement en syndicats et diverses organisations - se faire reconnaitre au côté du gouvernement (en co-gestion) le droit d'administrer et de contrôler l'accès et le partage du foncier entre agriculteurs.

S'il s'agit alors de reconstruire la France, il s'agit aussi de combler le retard des campagnes et de procéder à un vaste mouvement de modernisation agricole et rurale. Le modèle d'une petite agriculture familiale, fondé sur une main d'œuvre très nombreuse et faiblement productive (GERVAIS et al., 1965) s'était révélé en effet incapable d'assurer l'indépendance alimentaire du pays lors des trois crises qui se succédèrent de 1914 à 1945. La défaite (de 1940) attribuée au retard économique de la France, période qui avait vu la valorisation d'un agrarisme 
conservateur par le régime de Vichy, va durablement marquer la génération qui allait prendre en main la modernisation du pays et des campagnes. C'est à la lumière de ce contexte qu'il faut comprendre la véritable révolution qui allait suivre pour aboutir à ce que Mendras (1967) décrit comme la "fın des paysans”. La volonté modernisatrice portée par le gouvernement gaulliste au nom du "productivisme démocratique" (ALPHANDERY; SENCÉBÉ, 2009) va rencontrer les aspirations d'une jeunesse agricole formée par la Jeunesse Agricole Catholique (JAC) - soucieuse d'émancipation, de parité avec les urbains (en niveau et mode de vie), et de reconnaissance professionnelle. Il s'en suivra la cogestion des politiques agricoles entre la "profession" organisée autour des deux syndicats majoritaires (Centre National des Jeunes Agriculteurs, actuellement dénommé Jeunes Agriculteurs- CNJA, et Fédération Nationale des Syndicats d'Exploitants Agricoles - FNSEA) et le ministère de l'agriculture.

Cependant le modèle de l'exploitation familiale de taille moyenne - une fois modernisée - allait constituer le cadre jugé optimal de la modernisation. Précisons d'emblée que si la dimension familiale de ce modèle peut être défınie de façon assez aisée (une exploitation mise en valeur par 2 UTA - Unités de Travail Annuel- c'est-à-dire le couple associant ou pas les enfants), la taille dite “moyenne" fera l'objet de débats avant d'être encadrée, comme nous allons le voir, par des seuils qui feront eux même l'objet d'adapatation au fil du temps. S'agissant d'atteindre l'autonomie alimentaire au sein d'un pays relativement petit, l'objectif politique n'était pas d'établir de vastes entreprises agricoles avec salariés sur le modèle américain, mais de favoriser une agriculture intensive et productive. D'après Boinon (2011), ce choix avait deux raisons. L'une économique, tient à la rareté de la terre: l'exploitation moyenne ne peut jouer comme la grande exploitation sur une stratégie de rente foncière, et sera plus encline à réinvestir dans la production les gains de productivité attendus. La seconde raison est politique:

\begin{abstract}
pour obtenir un consensus sur une politique dont les effets prévisibles étaient la disparition d'une grande partie des agriculteurs, il fallait donner des garanties aux agriculteurs moyens que les terres libérées par les petits ne soient pas totalement récupérées par les plus gros agriculteurs ou par des personnes étrangères au monde agricole (BOINON, 2011, p.28).
\end{abstract}

La première pierre à l'édifice de la modernisation agricole fut le statut du fermage et du métayage de 1946. Véritable entaille au droit de propriété absolu hérité de la révolution française, ce statut va conférer au fermier de fortes garanties tout en donnant $\mathrm{au}$ foncier une nouvelle dimension, celle d'outil productif, en l'articulant à l'ancienne conception de "patrimoine privé". Le fermier devait être l'agent le plus actif de la modernisation étant assuré de se maintenir durablement (bail de 9 ans et renouvelable automatiquement), de pouvoir améliorer son outil de production par des investissements, d'en récupérer la valeur en cas de départ (dédommagement par le propriétaire), et finalement d'en acquérir la propriété (le fermier est "preneur" prioritaire en cas de vente) (DE CRISENOY, 1988).

Par ailleurs, en permettant aux métayers de demander la conversion de leurs contrats initiaux en bail à fermage, il condamnait à terme le métayage qui n'incitait guère les exploitants à augmenter leur production puisqu'une partie revenait au propriétaire. 
Ce nouveau statut fut une pièce maitresse de la modernisation car il garantissait la stabilité des structures foncières et limitait fortement la rente foncière en encadrant strictement les loyers (BOINON, 2011).

Mais, paradoxalement, cette faible rente foncière peut aujourd'hui, surtout aux abords des villes, alimenter la spéculation des propriétaires non exploitants, voire des agriculteurs à la retraite et in fine contribuer à l'urbanisation des terres agricoles et à l'augmentation des prix du foncier agricole (LEFEBVRE; ROUQUETTE, 2011). La stabilité foncière des fermiers, quant à elle, a constitué un moyen parfait de susciter chez ceux-ci les investissements nécessaires à la modernisation des structures (COURLEUX, 2011). Si ce statut a remis en question en partie le droit de propriété, c'était avant tout celui des propriétaires non exploitants afin d'en organiser le transfert vers les agriculteurs exploitants, jugé les plus aptes à opérer la transition vers une agriculture modernisée et fortement productive. Mais, l'effet recherché au départ par le législateur, favoriser l'accès à la propriété des exploitants, s'est soldé par un phénomène inverse: ainsi en 2010, près de 76\% de la Surface Agricole Utile (SAU) est exploitée en fermage (COURLEUX, 2011).

Ce phénomène s'explique selon Courleux (2011) par trois facteurs: démographique (allongement de la durée d'inactivité des retraités agricoles qui conservent souvent leur propriété foncière), sociologiques (faible activité de ces retraités sur le marché foncier) et structurels (concentration des exploitations et développement des formes sociétaires).

Le second facteur mérite d'être souligné: en effet la politique de modernisation orientée vers la sécurisation foncière des exploitants, mais aussi le mélange très particulier selon lequel la profession agricole s'est constituée en France, liant étroitement le travail et la famille. Muller (1984) explique cet attachement très particulier des agriculteurs retraités à leur patrimoine et ainsi le fait qu'ils ne le vendent pas de leur vivant.

Le troisième facteur constitue une rupture avec le modèle de l'exploitation familiale. A la très forte concentration des exploitations répond le morcellement des propriétaires, ce qui contribue à éloigner les premiers des seconds. Par ailleurs la constitution de formes sociétaires associées à des grandes tailles d'exploitations amène certains auteurs à s'interroger sur l'émergence d'une "agriculture de firme" (HERVIEU; PURSEIGLE, 2011) pas si éloignée, in fine, des modèles brésiliens.

La seconde pierre à l'édifice de la modernisation agricole, son ciment même (ALPHANDERY, 2001) fut la politique des structures initiée par les lois de modernisation agricole de 1960-1962, à l'origine de la politique dites des "structures". Celles-ci prévoient un vaste ensemble de mesures destinées à organiser le départ des "non modernisables” (paysans vieillissants, exploitants installés sur de trop petites structures pour être "viables") afin de libérer des terres pour conforter les structures aptes à suivre le chemin de la modernisation et installer de jeunes agriculteurs, formés aux nouveaux standards professionnels et porteurs $\mathrm{du}$ progrès technique. L'indemnité viagère de départ fut ainsi un outil d'accompagnement social de sortie de l'agriculture pour les premiers en même temps qu'elle permettait l'accès au foncier et/ ou aux exploitations pour les seconds.

Alors qu'au Brésil, un débat oppose actuellement les partisans néo-libéraux d'un traitement social de la "petite agriculture" à ceux qui défendent sa prise en compte comme segment agricole important et de- 
vant faire l'objet d'un traitement économique $^{11}$, en France, la question fut très vite tranchée. La "paysannerie" fut considérée sans hésitation comme relevant d'une politique sociale, pour permettre le transfert $\mathrm{du}$ foncier ainsi libéré vers les catégories cibles de la modernisation (jeunes et exploitants moyens) tout en humanisant les conditions de sa disparition.

D'autres mesures importantes vont encadrer la répartition du foncier à partir du modèle érigé par la Loi de 1960 et structuré autour de l'exploitation familiale de taille moyenne mise en valeur par deux Unités de Travail Annuel. La loi de 1962 va ainsi mettre en place deux seuils qui conditionnent l'accès au foncier dans la limite, jamais précisément définie, d'une "taille moyenne":

entre une superficie minimum de l'exploitation dont il est souhaitable d'éviter la disparition ou le démembrement et une superficie maximum au-delà de laquelle les cumuls et réunion d'exploitations ou de fonds agricoles, en vue de leur mise en rapport par un même exploitant doivent être soumis à autorisation préalable (Article 8).

Une Surface Minimale d'Installation sera définie en 1968 et orientera l'attribution du foncier et les diverses aides à l'installation. La commission départementale des cumuls, plus tard devenue commission des structures, va également encadrer l'agrandissement des exploitations. En effet l'exploitant, qu'il soit propriétaire ou fermier, doit obtenir une "autorisation d'exploiter" pour toute nouvelle terre acquise. A partir de ce moment, la propriété ne suffit plus, il faut l'aval de "la profession" et du gouvernement (représenté par le Préfet) qui animent cette structure. C'est sans doute la limite essentielle de cette politique qui repose en partie sur l'engagement de la profession localement (au niveau départemental) dans l'exercice d'un contrôle qui concerne ses propres rangs et qui suppose une forte sensibilité à l'enjeu de l'installation (BERNARDI; BOINON, 2009). En outre ce contrôle a sa propre faille, puisqu'il ne peut s'exercer sur les agrandissements familiaux: un fils s'installant à côté de son père et créant une société, une fois celui-ci parti en retraite, réunit finalement deux exploitations tout en passant entre les mailles du filet (BOINON, 2011).

La dernière pierre parachevant l'édifice est la mise en place des SAFER avec la loi de 1960 et son complément concernant le droit de préemption en 1962. Ces Sociétés d'Aménagement Foncier et d'Etablissement Rural succèdent à l'ancienne l'Association Nationale de Migration et d'Etablissement Ruraux (ANMER) présidée par Eugène Forget et qui sera le principal artisan de ces nouvelles structures. Ainsi, la curieuse référence, dans l'intitulé des SAFER, à la mission "d'Etablissement Rural” fait écho à la fonction première de son ancêtre qui consistait à rétablir l'équilibre entre "les terres sans homme et les hommes sans terre" (BOUCHU et al., 1999) dans un contexte où l'exode rural avait vidé certaines régions pauvres pendant

11. Ainsi, selon Alves et Rocha (2010), le groupe des plus pauvres (73\% des exploitations qui se partagent seulement 4\% de la VPB) ne devraient pas relever des politiques publiques de développement agricole, mais plutôt de politiques sociales. Considérant que ces pauvres ne sont pas de vrais agriculteurs, ils passent sous silence l'ensemble des politiques d'accès à la terre, qui n'ont aucun sens dans cette vision très libérale de l'agriculture. Selon cette perspective, le devenir de ces quelques 3,7 millions d'exploitations devrait se régler par l'exode rural, qui n'est qu'une question de temps. 
que d'autres étaient déjà touchées par une forte concentration et concurrence foncière (RENARD, 1969). Plus curieux encore est la proximité des termes "Etablissement" (dans le sens d'établir ou d'installer des hommes à la terre) et "Assentamento" (littéralement "établissement" dans le même sens) faisant référence à des zones divisées en plusieurs lots, situées sur le domaine foncier public ou sur des terrains expropriés et sur lesquelles ont été installées des familles sans terre à des fins d'agriculture (ELOY et al. 2002).

S'il s'agit en partie d'occuper des "terres sans hommes" par des "hommes sans terre", les "assentamentos" au Brésil ont été instrumentalisés durant la dictature militaire, à travers la politique de "colonisation", pour repousser vers les régions de frontière (Amazonie, Centre Ouest) les paysans pauvres, permettant ainsi de peupler et de contrôler ces marges brésiliennes sans remettre en question l'inégale répartition de la terre (ELOY et al., 2002).

Le rôle d'aménagement et de redistribution du foncier dévolu à la SAFER en France serait donc, comparativement, le point faible des politiques foncières au Brésil. En effet, la SAFER, outre son rôle stratégique dans la restructuration $d u$ foncier, a hérité très tôt d'un rôle d'aménagement: remembrement, drainage, aménagement forestier, opérations pour les collectivités ou l'Etat, faisaient ainsi partie de ses missions qui se sont élargies sans cesse jusqu'à en faire un "opérateur polyvalent de l'espace rural et périurbain" (SENCÉBÉ, 2012). En outre, cette société a été dotée de pouvoirs qui devaient en faire une pièce maîtresse du contrôle du foncier: sur l'information (notification par les notaires de tout projet de vente de terres agricoles), sur les transactions (droit de préemption), sur le marché (demande de révision du prix des terres mises en vente) et sur l'usage $\mathrm{du}$ foncier (choix des acquéreurs des terres rétrocédées). Mais son arme la plus puissante fut l'appui de la profession lui permettant un véritable "maillage territorial" que vient renforcer son organisation décentralisée à travers ses antennes départementales.

Cependant des limites, inhérentes aux dispositions légales qui organisent son action et aux évolutions plus globales de l'agriculture, affaiblissent son efficacité et remettent en question sa légitimité. Certains observateurs ont ainsi souligné les limites de leur capacité d'intervention: un champ d'action trop réduit (terres mises en vente librement) pour engager une réelle restructuration foncière et un droit de préemption sans cesse restreint (DUBY et WALLON, 1976, p. 232 et p. 610); l'obligation de revendre les terres acquises dans les cinq ans qui limite ses possibilités de location à des formules précaires ${ }^{12}$ et son système de financement par "revolving" (financement pour acheter en revendant) qui tend à favoriser le mieux disant des acheteurs (COULOMB, 1999).

Ainsi le fameux droit de préemption ne peut s'exercer si les acquéreurs appartiennent à la famille proche du vendeur ou s'ils sont "fermiers en place". Or, la structure de la propriété foncière relevant encore très largement des familles agricoles (y compris les héritiers ou bailleurs non exploitants eux-mêmes), une grande partie des transactions lui échappe sur un marché déjà très limité ${ }^{13}$, tandis que le

12. La SAFER ne peut ainsi qu'offrir des baux très précaires aux exploitants des terres qu'elle ne peut stocker que 5 ans (10 ans si c'est pour le compte d'une collectivité territoriale).

13. Les terres agricoles qui changent de propriétaires annuellement ne représentent que 1,3\% de la Surface Agricole Utile. 
fermage représente, nous l'avons vu, plus de 75\% de la SAU.

Mais des critiques sur son existence même ou sur sa gouvernance lui sont adressées comme le montre l'analyse des débats produits sur le forum "parlonsagriculture. com Le grand débat”, initié en 2009 par le ministère en charge de l'Agriculture ${ }^{14}$. Les auteurs de cette analyse, Antier et Marques (2011), réfèrent ainsi les différentes positions exprimées dans le débat notamment sur les politiques foncières et la SAFER à la typologie élaborée par Lacombe (2002). Audelà des "interventionnistes productivistes" qui défendent l'institution et les politiques foncières telles qu'elles ont été pensées, les critiques les plus radicales viennent du second groupe, "les libéraux productivistes", voyant en cette institution une entrave au libre marché et au droit de propriété.

De l'autre côté, les critiques portent moins sur l'existence d'un tel outil régulateur que sur son mode de fonctionnement d'une part, notamment son manque de représentativité syndicale, et de son orientation trop productiviste d'autre part. "Les interventionnistes multifonctionnels" peuvent être incarnés par la Confédération Paysanne qui défend l’idée d'une nécessaire intervention sur le foncier et l'agriculture mais aussi d'une ouverture des instances de régulation et d'une réorientation du modèle soutenu vers une agriculture multifonctionnelle.

Mais au-delà de ces différentes critiques, l'institution a du faire face au désengagement financier de l'Etat, l'obligeant ainsi à effectuer des choix "rentables" dans ses préemptions et rétroces- sions, mais aussi au désinvestissement de la profession. Ce qui constituait la "base sociale" de la SAFER, l'agriculture familiale de taille moyenne, s'affaiblit en effet sous l'effet de la fragmentation de la profession et de l'agrandissement tendanciel des exploitations. L'efficacité de son action était appuyée sur un réseau d'agriculteurs prêts à donner de leur temps pour en animer les instances et faire remonter du terrain toutes les informations nécessaires au suivi de la circulation des terres.

Ce réseau se débilite à mesure que l'individualisme et le modèle de la grande exploitation gagnent la profession. Dans ce contexte, l'institution s'est peu à peu tourné vers d'autres missions (développement local, urbanisation) et d'autres partenaires notamment les collectivités territoriales qui ont acquis de vastes compétences en matière foncière. Ces évolutions des deux dernières décennies pouvaient être ainsi interprêtées comme :

[le] passage d'un gouvernement du foncier organisé autour d'un projet politique agricole, cogéré par un État garant de l'intérêt général et par une profession unifiée à une situation de "gouvernances foncières locale" où coexistent des acteurs, des sources de légitimité et des projets multiples (SENCÉBÉ, 2012, p. 118).

Mais le vote en cours de la loi d'avenir de l'agriculture (2014) semble ouvrir de nouvelles perspectives avec le projet politique de refonder la politique agricole autour de l'agro-écologie ${ }^{15}$ et de renforcer les

14. Ce forum devait permettre d'alimenter la réflexion du ministère en vue de préparer la future Loi de Modernisation Agricole, promulguée en 2010.

15. Pour une présentation de cette loi voir notamment Búrigo; Cazella; Sencébé (2014). 
outils de contrôle du foncier dont l'efficacité avait été entaillée par des mesures libérales. La SAFER est ainsi dotée du pouvoir d'intervention sur les formes sociétaires. Reste cependant à voir comment, concrètement, ces mesures seront mises en œuvre, sachant que les organisations professionnelles agricoles qui en tiennent toujours les commandes n'ont pas vu leur gouvernance fondamentalement remise en question et que les lobbies de l'agro-industrie sont toujours influents.

\subsection{Au Brésil: le serpent de mer de la ré- forme agraire}

Il faut remonter à la période coloniale pour comprendre les inégalités actuelles qui marquent le Brésil tant du point de vue social que foncier. La priorité accordée, initialement par la couronne portugaise puis par les gouvernements brésiliens successifs, aux "grandes plantations " orientées vers l'exportation, explique en grande partie cette situation. Ces grands domaines perdurèrent au-delà de la fin de l'esclave, proclamée en 1888, grâce à la Loi des Terres de 1850. En effet, ce premier instrument juridique de régulation foncière stipulait que l'accès à la terre n'était possible que par son achat, ce qui permit à l'élite agraire de régulariser ses possessions coloniales durant les 38 années qui précédèrent l'abolition de l'esclavage. Comme le rappelle Martins (1981 et 2000):

cette loi eu pour objectif majeur de bloquer l'accès à la propriété foncière pour les travailleurs ruraux, spécialement les esclaves libérés et les immigrants européens, qui commençaient à être recrutés dans les grandes plantations de café du Sud Est.

Il fallut attendre les années 1960 pour que le thème de la réforme agraire soit mis à l'agenda politique avec les débats concernant le modèle national de développement ${ }^{16}$. En effet à partir du milieu des années 1950, le Nordeste du pays fut touché par une importante mobilisation politique de petits paysans avec pour épicentre les Ligues Paysannes, soutenues par le Parti Communiste Brésilien ${ }^{17}$. Mais le coup d'État militaire de 1964 va étouffer pour longtemps ces mouvements et réprimer durement toute opposition politique. La "réforme agraire", que le gouvernement militaire va mettre en place dès son arrivée avec le "Statut de la Terre"18, sera utilisée pour contrôler le mouvement social, l'affaiblir et déporter vers des territoires périphériques le problème foncier et les petits paysans sans terre. En effet, c'est à cette époque que fut créé l'Institut National de Colonisation et de Réforme Agraire (INCRA). La réforme agraire qui supposait

16. Les travaux de la Commission Economique pour l'Amérique Latine et les Caraïbes (CEPAL), organe de l'ONU, on aboutit à l'idée d'un développement “à partir de l'intérieur”, où l'insertion dans le marché externe est conditionnée à l'existence d'un marché interne fort et en expansion, ce qui présuppose la réalisation de la réforme agraire (GARCIA, 1998; SACHS, 1998).

17. Ce mouvement fut à l'origine de la création en 1963 de la Confédération Nationale des Travailleurs de l'Agriculture (CONTAG), qui reste actuellement l'une des principales forces syndicales de l'agriculture familiale. 18. Ce statut est inspiré de l'Alliance pour le Progrès du gouvernement des USA, qui craignait une extension de la révolution cubaine en Amérique Latine et qui faisait de la réforme agraire un moyen de limiter le risque d'embrasement social et révolutionnaire. 
"une meilleure distribution de la terre, au moyen de modifications dans le régime de propriété et d'usage, afin d'atteindre les principes de justice sociale et l'augmentation de la productivité" (BRASIL, 1964, Art. $1^{\circ} \S 1^{\circ}$ ), se solda finalement par une politique de colonisation des fronts pionniers comme l'Amazonie évitant de remettre en question les inégalités foncières. Ainsi rares furent les "désappropriations" de grandes propriétés ne remplissant pas la fonction sociale de la terre (du fait de leur sous occupation) comme le prévoyait le Statut de la Terre.

Le bilan chiffré de la politique de réforme agraire durant les gouvernements militaires s'avère, comme on pouvait s'y attendre, assez mince tout en étant difficile à établir sur le plan méthodologique, la transparence n'étant pas la première qualité des dictatures. En effet, au-delà de l'affichage d'un Statut de la Terre, les objectifs chiffrés que la réforme agraire devait atteindre n'ont pas été précisés dans les documents officiels. Par ailleurs, les données diffèrent selon les sources d'information. Enfin, les actions relevant de la réforme agraire à proprement dit n'ont pas toujours été distinguées des projets de colonisation.

Ainsi d'après Lima (2008), les installations réalisées durant la dictature, en prenant en compte la réforme agraire et la colonisation, n'ont concerné que 115.000 familles sur 13,5 millions d'hectares. Confirmant cette analyse, Marques, Del Grossi et França (2012) précisent que, durant cette période, seul 45.989 familles furent installées par la réforme agraire sur 9.490.709 ha. Ces chiffres correspondent à ceux qui figurent dans la base de données de l'INCRA qui intègrent les différentes politiques de réforme agraire qui se sont succédées jusqu'à aujourd'hui dans le pays. Fin 2011, selon cette base de données, 1.235.130 familles ont été installées par l'un des programmes de réforme agraire, ce qui signifie que celles qui en ont bénéficié durant la période militaire ne représentent que 3,7\% du total.

La préoccupation majeure du gouvernement militaire fut en fait le développement de politiques de modernisation de l'agriculture avec l'intention de constituer de grandes entreprises capitalistes. Contrairement à ce que proclamait le Statut de la Terre, c'està-dire comme en France, une répartition du foncier qui privilégie l'agriculture de taille moyenne par "l'extinction des latifundia et des minifundia”, le résultat fut un modèle de développement agricole marqué par les caractéristiques suivantes: l'augmentation de la concentration foncière, la priorité accordée à l'agriculture d'exportation, la révolution verte et l'exode rural (DELGAD0, 2010).

C'est ainsi une "modernisation conservatrice" qui se mit en place en transformant les bases technologiques de l'agriculture sans toucher à la structure foncière. L'enjeu prioritaire était alors de moderniser les latifundia et d'intégrer dans les principaux secteurs productifs certains segments de l'agriculture familiale en améliorant les infrastructures en milieu rural. Cette modernisation passa surtout par des politiques de crédit agricole subventionné et d'assistance technique, cependant mises en œuvre de façon très inégalitaire selon les régions et les types de producteurs.

Les régions Sud et Sud- Est qui en furent les principales bénéficiaires vont également constituer, paradoxalement, de nouveaux foyers de mobilisation politique des agriculteurs défavorisés. En effet, cette modernisation conservatrice va engendrer l'exclusion d'une grande majorité des petits agriculteurs qui ne réunissaient pas les conditions nécessaires (notamment la surface de leur exploitation) pour bénéficier de cette politique. Ainsi, contrairement à ce que l'on aurait 
pu attendre, le Mouvement des Sans Terre (MST) pris naissance en 1979 dans la région Sud et non pas au Nord-Est, pourtant très touché par la pauvreté. Selon Martin (2001, p. 25), le sud plus développé et urbanisé que le Nord a pu constituer un foyer militant plus important, d'autant qu'il s'y trouvait des ruraux urbanisés contre leur gré, formés et prêts à passer à l'action.

Mais une autre raison, historique, tient au processus de colonisation très particulier des trois Etats du Sud, marqué par l'installation d'agriculteurs immigrants d'origine italienne, allemande, polonaise et ukrainienne à partir du début $19^{\text {ème }}$. Selon Wanderley (1999), ce modèle de colonisation représente un des foyers constitutifs de l'agriculture familiale brésilienne. Ainsi, le développement de l'agriculture dans la région Sud n'a pas suivi le modèle classique de la grande plantation marquant le reste du pays. Les agro-industries ont développé, avec l'aide de l'Etat, d'étroites relations avec les unités agricoles familiales localisées dans le Sud. Le système de l'intégration verticale de porcs, volailles et, plus tard, de lait et de tabac en fut la forme prédominante.

Cette intégration a la particularité de s'être effectuée avec le soutient actif de l'État et d'avoir entrainé de forte inégalités, comme l'a bien montré Martine (1991, p. 35) pour qui le processus fut "partiel, inégal, fortement soutenu par les ressources publiques, en soi limité, et relevant davantage d'un capitalisme d'État que d'un capitalisme moderne".

L'instabilité des agriculteurs non propriétaires ou des très petits propriétaires engendra des formes de reproduction sociale des plus variées. L'exode rural ou le salariat agricole furent des alternatives choisies par certains, mais la recherche de terre incessante pour continuer à cultiver fut, sans conteste, l'option que prit la majorité d'entre eux, ceux-là même qui allaient constituer, selon les conjonctures politiques, des acteurs de la lutte pour la terre.

La fin des années 1970 marque le début de l'ouverture politique et de la "transition démocratique", mettant fin à deux décennies de dictature militaire avec la montée en puissance de la société civile. En ville comme à la campagne des espaces d'expression populaire virent le jour et le parti unique d'opposition remporta victoire sur victoire. Dans ce contexte de changement politique et social, la question agraire revint à l'ordre du jour: l'exclusion économique dont était victime la paysannerie fut l'une des raisons de son émergence en tant qu'acteur politique.

L'idée de réforme agraire et de politiques publiques orientées vers l'agriculture familiale commença à gagner du terrain au sein même de l'Etat. Dans les campagnes, le Mouvement des Sans Terres surprit l'élite agraire en occupant des fazendas - grands établissements ruraux avec un faible indice de productivité agricole - pour les agriculteurs sans terre dans la région Sud parallélement à la forte mobilisation politique pour faire approuver le $1^{\text {er }}$ Plan National de Réforme Agraire de la Nouvelle République (I PNRA) qui succéda à la dictature (1985). Ce plan prévoyait de “désapproprier" 43 millions d'ha devant bénéficier à 1,4 million de familles en 5 ans (1985-1989). Mais les résultats furent très en-deçà des objectifs, avec seulement 89900 familles installées sur $6,4 \%$ des surfaces prévues. D’après Condé $(2006$, p. 49) ce très faible résultat fut la "conséquence directe des pressions contraires exercées par les forces politiques des grands propriétaires de terre, qui, en réaction à l'émergence des mouvements sociaux, formèrent l'Union Démocratique 
Ruraliste (UDR) ${ }^{19}$, en 1985”. Ce mouvement politique d'extrême droite a su évoluer vers la constitution d'un groupe de parlementaires fédéraux pluri-partis, qui s'oppose systématiquement à l'adoption de mesures favorables à la réforme agraire ${ }^{20}$. Son principal leader est une sénatrice qui préside la Confédération Nationale de l'Agriculture et qui se revendique elle-même de l'agrobusiness brésilien.

Le contexte actuel reste marqué par la polarisation en deux grands fronts politiques, qui se font représenter à l'intérieur de l'État (fédéral). D’un côté les divers mouvements, syndicats et organisations de la société civile trouvent dans le MDA leurs principaux moyens d'intervention et d'accès aux politiques publiques dédiées à l'agriculture familiale et aux "populations traditionnelles” (amérindiens, quilombolas, ribeirinhos, extrativistas, etc. ${ }^{21}$ ). L'INCRA a été rattaché à ce Ministère et reste l'organisme responsable de l'exécution de la réforme agraire, de la structuration et de l'assistance aux "assentamentos” des agriculteurs sans terre.

À l'intérieur du MDA, trois secrétariats sont été créés: le Secrétariat de l'Agriculture Familiale (SAF), le Secrétariat de Développement Territorial (SDT) et le Secrétariat de
Réorganisation Agraire (SRA). Le premier dirige le principal programme de ce Ministère - le Programme National de Renforcement de l'Agriculture Familiale (PRONAF), le deuxième le Programme National de Développement Territorial Durable (PRONAT) et le troisième le Programme National de Crédit Foncier (PNCF). De l'autre côté, les grandes exploitations agricoles, principalement orientées vers l'exportation ont le Ministère de l'Agriculture, de l'Élevage et de l'Approvisionnement (MAPA), comme principal interlocuteur et source de financements publics dédiés à l'agrobusiness. Cependant leur pouvoir n'est pas équivalent comme le montre la disproportion des ressources publiques allouées à chacun des deux ministères. Ainsi, pour la campagne agricole de 2012-2013, le MDA ne peut compter que sur 18 milliards de Reais, alors que le MAPA dispose de 107 milliards. Ces chiffres montrent les profondes différences structurelles et politiques existant entre les deux grands pôles de l'agriculture brésilienne. Il faut cependant mettre en regard ce déséquilibre avec les montants alloués en France aux aides liées aux premiers et seconds piliers de la $\mathrm{PAC}^{22}$, les premières

19. Cette entité, outre les méthodes traditionnelles de répression et de violence contre les travailleurs ruraux, s'est approprié le discours sur la libre initiative et la propriété privée pour s'assurer des alliances politiques avec les entrepreneurs du secteur industriel et financier (MEDEIROS, 2002).

20. Ces différents partis peuvent apparaitre comme modérés et ouverts, mais ils sont financés par des banquiers et des industriels qui sont eux-mêmes de grands propriétaires terriens, ce qui illustre le pouvoir encore réel de l'oligarchie foncière brésilienne.

21. Ainsi, les Quilombolas sont des descendants d'esclaves ayant fuit les fazendas à l'époque esclavagiste pour occuper des terres dans des zones très éloignées. Leurs descendants qui y résident toujours, demandent au gouvernement la légalisation des terres qu'ils occupent. Les Ribeirinhos sont des agriculteurs-pêcheurs qui vivent en bordures des rivières dans l'Amazonie. Ils pratiquent une agriculture de subsistance complétée par la pêche. Les Extrativistas vivent également en forêt, de l'exploitation des ressources naturelles, comme l'extraction du caoutchouc, la cueillette de fruits et d'herbes médicinales, etc.

22. Le premier pilier concerne les "aides directes" aux producteurs en soutien à la production et sont destinées notamment à compenser le différentiel des prix entre le marché mondial et le marché intérieur (soutien donc aux exportations). Le second pilier concerne des politiques structurelles: développement rural, modernisation, installation, reconversion, protection environnementale. 
bénéficiant aux plus grandes structures et englobant 87\% du total des aides versées.

$\mathrm{Au}$ fınal, l'intervention de l'État brésilien sur la structure foncière est passée, et passe toujours, par trois types de politique: la réforme agraire à travers la désappropriation de terres, la régularisation foncière et, enfın, le crédit foncier.

Le premier cas concerne l'installation d'agriculteurs sans terre sur des grands domaines ainsi “expropriés". Cette politique, prévue dès 1965 nous l'avons vu, n'a été réellement mise en œuvre qu'à partir de 1985 avec la ré-démocratisation du pays et les pressions du MST. L'INCRA est chargé de défınir les aires expropriables en fonction $d u$ degré d'utilisation de la terre dans les exploitations concernées, en constatant si, oui ou non, la terre remplit sa fonction sociale" et si les zones concernées permettent l'installation "viable" de petits paysans (infrastructure, accessibilité...). Le MST anticipe très souvent l'action de l'INCRA en occupant les domaines ainsi potentiellement expropriables, pour faire pression sur le gouvernement et le mettre devant le "fait accompli".

La régularisation foncière constitue une reconnaissance officielle par le gouvernement fédéral du statut de propriétaire aux “occupants" jusque-là sans titre de propriété. Les terres concernées relèvent de "réserves foncières” de l'Etat, destinées à la colonisation pour de petits paysans ou à l'installation des grandes entreprises investissant dans les régions concernées. Cette politique a une forte incidence surtout en Amazonie où la politique de colonisation a favorisé les situations d'occupations précaires. Ce genre de politique est fortement critiqué par le MST pour lequel la régularisation foncière ne peut être assimilée à la réforme agraire, dans le sens où elle ne remet pas en question la structure foncière contrairement aux désap- propriations foncières. Cependant, la légalisation des terres occupées de façon précaire constitue un enjeu important puisque c'est seulement à partir de l'obtention d'un titre de propriété que les agriculteurs peuvent accéder aux autres politiques, notamment le Pronaf. Or la situations “d'occupants” précaires concerne 412.357 familles d'après le dernier recensement agricole. Par ailleurs, le titre de propriété offre une certaine sécurité foncière aux agriculteurs dans un contexte où les pressions ne sont pas rares pour s'approprier, par la force, les terres sans "propriétaires officiels". Au-delà de ces débats, le bilan chiffré de ces deux politiques (entre 1985 et 2009) montre que les actions de régularisation foncière ont bénéficié à $20 \%$ des familles installées et 57\% de la surface acquise dans le cadre de la réforme agraire; tandis que les désappropriations ont concerné 63\% des familles sur 31\% des terres de la réforme agraire. La localisation des premières, situées le plus souvent sur des zones de colonisation, explique que les surfaces attribuées soient plus importantes que celles dont bénéficient les familles installées par désappropriation de domaines situés le plus souvent dans les régions anciennement défrichées et plus peuplées.

Enfin, le Programme National de Crédit Foncier, initié en 2003, constitue une adaptation de deux programmes antérieurs (Cédula da Terra et Banco da Terra) créés dans les années 1990. Leur principe est de permettre aux petits agriculteurs familiaux d'accéder à la propriété par le marché au moyen du crédit. Le gouvernement avance ainsi les sommes nécessaires à l'achat que les agriculteurs remboursent sur une longue échéance avec des taux bonifiés. La portée de ces différents programmes reste cependant encore limitée avec 131.552 familles bénéficiaires au total fin 2010. Deux 
facteurs principaux peuvent expliquer leur faible développement: des résistances d'ordre idéologique, et l'absence de tradition et de base technique des principales agences publiques chargées de leur mise en œuvre (CAZELLA, 2010).

La politique de crédit foncier est confrontée à une forte résistance des organisations impliquées dans la lutte pour la terre, cellesci la considérant en effet comme un moyen de démobilisation politique des mouvements sociaux. Néanmoins, certains secteurs du syndicalisme lié à l'agriculture familiale, notamment la Confédération Nationale des Travailleurs de l'Agriculture et plus récemment la Fédération Nationale des Travailleurs de l'Agriculture Familiale (FETRAF) ${ }^{23}$, en sont venus à reconnaitre la nécessité et les possibilités d'actions complémentaires dans le domaine de la réforme agraire. Selon cette perspective, la politique de crédit foncier est considérée comme un instrument qui permet de sauvegarder le caractère familial de l'agriculture, en assurant la reprise des exploitations familiales mises en vente (sans successeur notamment), en augmentant la taille des "minifundio" et en contribuant à la restructuration des unités de production agricole. Dans les Etats de colonisation ancienne, le crédit foncier s'avère tout à fait adapté, car les frontières sont "épuisées" 24 et la désappropriation foncière à des fins de réforme agraire se confronte aux limites imposées par la législation. Par ailleurs, le crédit foncier offre également une solution aux très nombreuses exploitations agricoles familiales sans successeur, souvent rachetées par des investisseurs locaux pour être transformées en plantations forestières d'espèces exotiques ou pour être intégrées dans de grandes exploitations capitalistes. Dans les Etats où subsiste encore une frontière, comme dans le Piauí ${ }^{25}$ où nous avons enquêté, le crédit foncier permet de stabiliser des familles jusque-là enchainées à des travaux précaires de tâcherons sur de grandes plantations. Les trois assentamentos étudiés ont bénéfıcié du crédit foncier articulé d'autres politiques (Faim Zéro et le Programme d'acquisition d'aliments dédiées aux régions pauvres) obligeant d'une part les candidats à s'organiser en association pour former un assentamento collectif et assurant d'autre part le rachat d'une partie de la production par l'État pour sa mise à disposition pour la restauration collective locale (écoles, hôpitaux...). Cette organisation collective a plusieurs avantages: le crédit foncier donne ainsi tous ses effets en termes d'amélioration des conditions de vie soulignée par tous les bénéficiaires rencontrés, car la "collectivité” participe dans un premier temps à la construction ou à la rénovation de l'habitat et de certaines infrastructures (eau, salle commune, irrigation...). Par ailleurs la partie collective de la production facilite sa mise en vente sur le marché (quantité et complémentarité des produits) tandis que la pro-

23. La FETRAF est un syndicat spécialement dédié à l'agriculture familiale (elle ne prend pas en charge les salariés agricoles comme la CONTAG) et qui a été reconnu récemment. La Fédération se positionne plus à gauche que la CONTAG.

24. Au sens où il n'y a plus de fronts pionniers à coloniser, ni de nouvelles terres à défricher.

25. Le Piaui est l'un des États les plus pauvres du Brésil. Il constitue une zone de transition entre l'Amazonie et le "sertão" très sec du Nordeste. Peu peuplé, il est également très peu développé au niveau économique et ne bénéficie pas du “décollag” économique récent marquant certains États du Nord-Est. 
duction familiale sert toujours à l'autoconsommation. Mais surtout, la constitution en collectif donne aux paysans un certain poids dans les négociations entre l'État et les propriétaires (fixation du prix du foncier que l'État rachète puis revend aux paysans) et sécurise au quotidien leur présence dans des zones souvent reculées.

\section{Conclusion: enseignements et regards croisés}

En France, l'exploitation familiale de taille moyenne a constitué la base sociale et le modèle de développement sur lequel se sont appuyées la modernisation agricole et les politiques foncières. De véritables mesures - contrairement au Brésil - ont été prises pour en finir "avec les latifundia et les minifundia” selon les termes du Statut de la Terre qui en faisait aussi l'objectif jamais réalisé - de la réforme agraire. Le facteur expliquant ici sa mise en œuvre et là son ajournement perpétuel tient selon nous à la structure sociale et politique des deux pays. La convergence des élites agraires issu de la JAC - et des élites gouvernementales - gaullistes modernisateurs - a été à l'origine de la politique des structures. Cette convergence a été rendue possible par des effets de génération (partage de l'expérience de la guerre et de la résistance) et de concomitance des aspirations socio-économiques de la jeunesse agricole et des objectifs de politique économique du gouvernement. $\mathrm{Au}$ Brésil la structure du pouvoir économique et politique étant marquée par la prééminence des grands propriétaires terriens, aucune convergence ne fut possible entre les intérêts défendus au niveau gouvernemental et les revendications des mouvements sociaux. L'histoire économique et la politique agraire du Brésil se sont finalement construites sur la dualité de l'agriculture: le système lati- fundiaire où les esclaves travaillaient pour leurs maitres, n'a pas totalement disparu dans les faits. La fin de l'esclavage, préparé par la loi sur la terre, s'est traduite par la "libération" d'un grand nombre de futurs paysans sans terre qui allaient constituer la réserve de travailleurs des plantations. Les grands propriétaires fonciers ont joué de leur influence politique pour maintenir à l'état de projet la réforme agraire. Dans le Brésil de Lula, bien que la politique menée ait considérablement amélioré la situation des plus pauvres et contribué à élargir la classe moyenne, en matière agricole, la dualité demeure. Elle semble organisée de façon complémentaire entre l'agriculture d'exportation qui fournit au pays les devises nécessaires à son développement et l'agriculture familiale qui nourrit sa population. Cette dualité se retrouve au sein même de l'agriculture familiale dont une partie non négligeable produit pour l'exportation. Au-delà de cette dualité, se maintient une très forte diversité et inégalité des statuts fonciers entre les différents types d'agriculteurs.

Ce que mettent en lumière ces histoires contrastées, c'est que la politique foncière est un levier puissant agissant sur l'attribution et de la redistribution des richesses et des opportunités à l'échelle d'un pays. Toute réforme agraire, toute restructuration foncière constitue une remise en cause d'un ordre existant: la structure foncière est avant tout une structure sociale soutenue, maintenue, ou redéfinie par un pouvoir politique. Cette remise en question est passée en France par la convergence - finalement assez contingente - des intérêts de l'élite politique avec ceux d'une partie de la profession à un moment donné de l'histoire socio-économique du pays. Au Brésil, elle repose sur la lutte des mouvements sociaux liés à la terre et sur des actions radicales (occupation) visant à faire 
pression sur le pouvoir politique pour faire évoluer la structure agraire au détriment de l'élite foncière. Peut-on dire alors que le modèle français est plus solide que le brésilien, s'il repose sur l'heureuse rencontre entre certains intérêts à un moment donné de son histoire, par ailleurs de plus en plus lointaine?

La politique des structures a eu pour effet une très forte sécurité et stabilité foncière des exploitants ce qui a permis la mise en place d'une agriculture très productive par de forts investissements dans l'appareil productif au détriment de la rente foncière étroitement contrôlée et limitée.

En définissant les droits qui forment une quasi "propriété culturale" de la terre, le statut du fermage peut être vu comme une politique qui assure la coexistence entre la logique économique du fermier et la logique patrimoniale du bailleur par une répartition équilibrée des droits de propriété entre ces deux acteurs (COURLEUX, 2011, p. 51-52).

Mais jusqu'à quand l'attachement patrimonial des propriétaires assurera-t-il le maintien de la terre aux mains des agriculteurs, dont les familles s'éloignent de l'agriculture à mesure que le groupe lui-même se rétrécit? Par ailleurs, l'atomisation de la propriété a pour corolaire la concentration des structures d'exploitations et l'émergence d'une agriculture se rapprochant du modèle de la firme. Ces sociétés de plus en plus capitalistiques posent la question de leur transmission, et l'appel à des "apports de capitaux" pour l'installation des jeunes agriculteurs, de plus en plus fréquent, amène à s'interroger sur l'engagement de ce genre d'investisseurs sur le long terme, d'autant que la terre devient un placement qui peut rapporter...

Mais c'est aussi la mise en place d'une "méritocratie foncière" qui a réglé l'accès à la terre de façon à la fois endogame et sélective, excluant à chaque génération les "moins viables ou modernisables" de la profession et ouvrant le chemin à l'agrandissement tendanciel des exploitations. La politique des structures contenait donc en elle à la fois la promotion et la ruine de l'agriculture familiale de taille moyenne. Cette centrifugeuse agraire, qui a pour fil la fin des paysans, génère ainsi ses propres contradictions mais alimente à chaque époque des résistances et des retours paysans, du Larzac des années 1970 aux agriculteurs se réclamant d'un modèle plus durable aujourd'hui (AUCLAIR et al., 2006). Ces retours et résistances s'exercent néanmoins, jusqu'à présent, et comme au Brésil pour les mouvements sociaux liés à la terre, par des formes contestataires et par des groupes n'ayant pas ou peu accès au pouvoir sur le foncier et aux différents organes régulant la politique agricole, ainsi que l'incarne la Confédération Paysanne en France et ses homologues brésiliens, la Fédération Nationale des Travailleurs de l'Agriculture Familiale en tant que syndicat de défense de la petite agriculture familiale et le MST en tant que mouvement social engagé dans le lutte pour l'accès à la terre.

$\mathrm{Au}$ Brésil, la politique foncière n'a pas été, comme en France, mise en place à partir d'un socle unifié et d'un modèle agricole clairement identifié, elle est plutôt le résultat historique des rapports de force entre élites agraires et mouvements sociaux. Les trois types de leviers évoqués plus haut (expropriation, régularisation, crédit foncier) en constituent ainsi des strates à la fois sédimentées et refaçonnées à chaque époque. Actuellement, chacun de ces leviers fait l'objet de critiques fortes de la part des défenseurs et membres de l'agrobusiness mais aussi de débats entre les partisans d'une 
meilleure répartition foncière (mouvements sociaux, universitaires, membres du MDA, syndicats). Ainsi, la régularisation foncière, contestée par le MST comme un moyen de masquer l'absence de redistribution foncière, est défendue par les plus pragmatiques comme un moyen de sécurisation foncière des petits paysans. L'expropriation foncière, défendue par le MST comme moyen de pression pour faire avancer le processus de réforme agraire, est disqualifié par les organisations professionnelles de l'agrobusiness sous le pretexte de la précarité auxquelle elle expose les familles durant le processus qui va de "l'assentamento" à l'attribution du titre de propriété. Enfin le crédit foncier, défendu par les plus pragmatiques comme moyen de sécurisation foncière, de renouvèlement des exploitations familiales et d'amélioration des conditions de vie paysanne, est mis à l'index par le MST qui y voit une solution marchande et non politique aux inégalités agraires et un instrument de pacification sociale. En face, malheureusement, l'élite agraire semble unie face à l'enjeu de maintenir sa position foncière, sociale et son influence politique. Certaines évolutions récentes, notamment la position favorable du CONTAG et de la FETRAF vis-à-vis du crédit foncier, laisse cependant entrevoir des convergences possibles entre différents segments sociaux, condition qui semble favorable à l'articulation des différents leviers fonciers dans la perspective d'une avancée de la réforme agraire.

Finalement à partir de cheminements très différents, c'est une tendance à la dualité de l'agriculture qui s'observe en France comme au Brésil. Cette dualité ne recouvre pas les mêmes réalités sociales et économiques en termes d'effectifs concernés, de niveau d'inégalités sociales entre les deux pôles mais elle met en jeu les mêmes mécanismes de concentration foncière et les mêmes difficultés d'accès à la terre pour les petits agriculteurs. L'antériorité du Brésil en matière de dualité de son agriculture pose en tout cas certaines questions concernant les champs $\mathrm{du}$ futur français: assisterons-nous à une dualité complémentaire entre l'agriculture d'exportation et l'agriculture de proximité et de qualité? Mais en France, contrairement au Brésil, la petite agriculture ne concerne que très peu d'effectifs et de consommateurs solvables (ce qui est rare est cher!) et ne saurait pour l'instant suffire à assurer la souveraineté alimentaire. En l'absence d'un soutien fort à ce type d'agriculture et d'une politique foncière pour faciliter son accès à la terre, ne risque-t-on pas d'assister à d'autres formes de dualité: celle des consommateurs ayant accès ou pas aux produits de qualité et de proximité, celle des paysages banalisés par la grande agriculture céréalière d'un côté et mis en valeur voire en folklore par une agriculture "paysagère" de l'autre, celle enfin de l'environnement préservé dans des zones sanctuarisées au milieu de vastes ensembles gérés par une agriculture, au mieux, "raisonnée"? Car, au-delà de la structure foncière, c'est bien de la société plus largement dont il s'agit: de son modèle agricole, de son alimentation, de la gestion de la ressource naturelle que constitue la terre et donc de la préservation de l'environnement.

\section{Références}

AGRESTE PRIMEUR. Les premières tendances du recensement agricole de 2010, no 272, sept. 2011.

AGRESTE PRIMEUR. L'utilisation du territoire entre 2006 et 2009, nㅡㄴ 246, juin. 2010.

AGRESTE PRIMEUR. La maison individuelle grignote les espaces naturels, no 219, jan. 2009. 
ALPHANDERY, P. Les campagnes françaises de l'agriculture à l'environnement (1945 -2000): politiques publiques, dynamiques sociales et enjeux territoriaux. 2001, 465 f. Thèse (Doctorat en Sociologie). FNSP, Fondation Nationale des Sciences Politiques, Institut d'Etudes Politiques de Paris, Paris. 2001.

ALPHANDERY, P.; SENCÉBÉ, Y. L'émergence de la sociologie rurale en France (1945-1967). In: Etudes rurales, jan / juin, no 183, p. 23-40. 2009.

ALVES, E.; ROCHA, D. de P. Ganhar tempo é possivel? In: Gasques J. G., Vieira Filho J. E. R., Navarro Z. (Orgs.), A agricultura brasileira: desempenho, desafios e perspectivas. Brasília, IPEA, p. 275-290. 2010 ,

ANTIER, C.; MARQUES P. E. M. Concepções e modelos agrários em concorrência na gestão fundiária da França: questões oportunas para refletir sobre o caso brasileiro, Revista Política Pública, Vol. 15, no 1, p.13-20. 2011.

AUCLAIR, L.; ASPE, C.; BAUDOT, P. (Dir.). Le retour des paysans? À l'heure du développement durable, Paris: IRD Editions, 2006.

BERNARDI, V.; BOINON, J-P. L'action des syndicats agricoles en faveur de l'installation dans les commissions départementales d'orientation agricole, Économie rurale, ํㅜ 312, p. 80-92. 2009.

BERNIER, M. Évolution de la démographie agricole et ses conséquences sur l'organisation, le fonctionnement et la transmission des exploitations agricoles. Rapport de mission parlementaire auprès du Ministre de l'agriculture et de la pêche., La documentation Française, décembre 2005.

BOINON, J..P. Les politiques foncières agricoles en France depuis 1945. Économie et Statistiques, no 444-445, p. 19-37. 2011.

BOUCHOU, H. et al. Partager la terre - l'histoire des Safer, Ed. Atlântica, 1999.

BRASIL. Estatuto da Terra, Brasília, 1964.

BÚRIGO, F. L. ; CAZELLA, A. A. ; SENCÉBÉ, Y. A "lei do futuro": novos rumos para a agricultura francesa ? Bolletim do OPPA, ㄲo 45, mar. 2014.
CAZELLA, A. A.; BÚRIGO, F. L. 0 Plano Brasil Sem Miséria não contempla as especificidades da pobreza rural. Boletim do OPPA, no 38, jul., 2011. Disponivel em <http://oppa.net.br/artigos/ portugues/artigo_0PPA_br_038-07_2011>. Acesso em: 28 ago. 2012.

CAZELLA, A. A. Crédito fundiário: reforma agrária de mercado ou instrumento salvaguarda da agricultura familiar? Boletim do OPPA, no 34 , jun. 2010. Disponivel em $<\mathrm{http}: / /$ oppa. net.br/artigos/portugues/artigo_0PPA_br_03406_2010>. Acesso em: 28 ago. 2012.

CAZELLA, A. A.; BONNAL, P.; MALUF, R. S. Agricultura familiar: multifuncionalidade e desenvolvimento territorial no Brasil. Rio de Janeiro: Mauad X, 2009.

CGDD (Commissariat Général du Développement Durable). L'artificialisation des sols s'opèrent au détriment des terres agricoles, Le point Sur. Février, 2011.

CONDÉ, P. A. Acesso à terra e a visão do público não mobilizado em acampamentos: o crédito fundiário do município de Caçador. Dissertação (Mestrado em Agroecossistemas). Centro de Ciências Agrárias - UFSC, São Carlos-SP, 2006.

COULOMB, P. La politique foncière agricole en France: une politique foncière à part? ", Cahiers options méditerranéennes, v. 36, p. 69-94. 1999.

COURLEUX, F. Augmentation de la part des terres agricoles en location: échec ou réussite de la politique foncière? Économie et Statistique no ${ }^{-}$444445, p.39-53. 2011.

DE CRISENOY, C. De l'origine et du rôle de la politique foncière agricole, Économie Rurale, ํㅜㅁ 184185-186, p. 85-91. 1988.

DUBY, G.; WALLON, A. Histoire de la France rurale de 1914 à nos jours, T. 4, Paris: Seuil, 1976. DELGADO, N. G. 0 papel do rural no desenvolvimento nacional: da modernização conservadora dos anos 1970 ao governo Lula. In: MOREIRA, R. J.; BRUNO, R. (Orgs.). Dimensões rurais de políti- 
cas brasileiras, Rio de Janeiro: Mauad X; Seropédica, RJ: Edur, 2010, p.17-53.

ELOY, L. et al. Questions foncières et politiques de réforme agraire au Brésil. Géoconfluence - Dossier: le Brésil, ferme du monde, 2002. [En ligne: consulté le 2/09/12].

GARCIA, A. La construction interrompue: Celso Furtado, la guerre froide et le développement du Nordeste, Actes de la Recherche en Sciences Sociales, v. 121-122, p. 52-61. 1998.

GERVAIS, M.; SERVOLIN, C.; WEIL, J. Une France sans paysans. Paris: Seuil, 1965.

GILLET, M. Analyse sociologique des transmissions dites hors cadre familial des exploitations agricoles, Economie rurale, no 253, p. 87-90. 1999.

HERVIEU, B.; PURSEIGLE, F. Des agricultures avec des agriculteurs, une nécessité pour l'Europe, Projet, no 321, avr.-mai, p. 60-69. 2011.

HERVIEU, B.; VIARD, J. L'archipel paysan. La fin de la république agricole. La Tour d'Aigues: Ed. de l'Aube, 2001.

IBGE. Censo Agropecuário de 2006, IBGE, Brasília, 2006.

IGAS. Pauvreté, précarité, solidarité en milieu rural, Rapport de l'Inspection Générale des Affaires Sociales et du Conseil Général de l'Agriculture, de l'Alimentation et des Espaces Ruraux, Septembre, Tome I. 2009.

LACOMBE, P. L'agriculture à la recherche de ses futurs, La Tour d'Aigues/Paris: Ed. de l'Aube/ Datar, 2002.

LEFEBVRE, L.; ROUQUETTE, C. Les prix du foncier agricole sous la pression de l'urbanisation, Economie et Statistique, no 244-245, 2011, p. 155-180.

LIMA, F. Programa cédula da terra: uma releitura dos principais resultados. Dissertação (Mestrado em Desenvolvimento Econômico], UNICAMP, Campinas, 2008.

MARQUES, V. P. M. A.; DEL GROSSI, M. E.; FRANÇA, C. G. 0 censo 2006 e a reforma agrária: aspectos metodológicos e primeiros resultados.
Brasília: Ministério do Desenvolvimento Agrário, 2012.

MARTIN, J-Y. Les sans-terres du Brésil - Géographie d'un mouvement socio-territorial, L'Harmattan. 2001.

MARTINE, G. A trajetória da modernização agrícola: a quem beneficia? Lua Nova, no 23, São Paulo: Marco Zero, 1991.

MARTINS, J. de S. Os camponeses e a política no Brasil, Petrópolis, RJ: Vozes, 5. ed., 1981.

MARTINS, J. de S. Reforma agrária - o impossivel diálogo sobre a História possível. Tempo Social. Revista Sociológica, USP, São Paulo, no 11(2), p. 97-128. 2000.

MEDEIROS, L. Movimentos sociais, disputas políticas e reforma agrária de mercado no Brasil, Rio de Janeiro: Edur/Unrisd, 2002.

MENDRAS, H. La fin des paysans. Paris: Armand Colin, 1967.

MULLER, P. Le technocrate et le paysan. Essai sur la politique française de modernisation de l'agriculture. Paris: Ouvrières, 1984.

NUNES, S. P. Censo Agropecuário demonstra aumento das escalas de produção e redução das ocupações agrícolas. Curitiba: Deser, Boletim Eletrônico, nº 164. 2008.

RENARD, J. Les Migrations rurales des Vendéens vers le sud-ouest: Un phénomène moribond? Norois, ํㅡㅇ 62 p. 177-189. 1969.

SACHS, I. L'imagination et le savoir: le développement selon Furtado, Cahiers du Brésil contemporain, p. 179-183. 1998.

SCHNEIDER, S.; CAZELLA, A. A.; MATTEI, L. F. Histórico, caracterização e dinâmica recente do Pronaf - Programa Nacional de Fortalecimento da Agricultura Familiar. In: SCHNEIDER, S.; SILVA, M. K; MARQUES, P. E. M. (Org.). Políticas Públicas e participação Social no Brasil Rural, Porto Alegre: UFRGS, v.1, p. 21-49. 2004.

SENCÉBÉ, Y. La SAFER - De l'outil de modernisation agricole à l'agent polyvalent du foncier: 
hybridation et fragmentation d'une institution, Terrains et Travaux, 2012/1 - no 20, p. 105-120. 2012.

SENCÉBÉ, Y. (Org.) Rapport final programme PSDR Inra Bourgogne Teragri, 2011, 46p.

WANDERLEY, M. N. B. Raizes históricas do campesinato brasileiro, In: TEDESCO J. C. Agricultura familiar: realidades e perspectivas. Passo Fundo, EdUPF, p. 23-56. 1999. 


\section{RESUMÉ}

Cet article propose une mise en regard de deux pays, la France et le Brésil, où l'agriculture tient une place particulière, avec cependant des trajectoires et des enjeux contrastés. L'analyse est centrée sur la question foncière. Les données sont issues de travaux de terrain menés en parallèle et en commun et de la lecture croisée de sources statistiques et bibliographiques concernant l'histoire et les politiques foncières et agricoles des deux pays. L'étude, en première partie, des enjeux fonciers fait apparaitre une tendance à la dualisation de l'agriculture dans chacun des deux pays à partir de cheminement très contrastés. Au Brésil de très petites exploitations en situation de pauvreté et d'insécurité foncière voient leur nombre augmenter plus vite que la part du "gâteau" foncier et économique qui leur est réservé. En France, toujours moins d'exploitations toujours plus grandes accaparent l'essentiel des terres libérées dans un contexte de forte concurrence foncière. La remontée dans l'histoire des politiques foncières et agricoles, menée en seconde partie, montre qu'au-delà des objectifs similaires affichés de deux côtés de l'Atlantique, seul la France a mis en place une véritable restructuration foncière au profit de l'agriculture familiale de taille moyenne qui a constitué le socle de la modernisation agricole. Au Brésil, la politique foncière est plutôt le résultat historique des rapports de force entre élites agraires et mouvement sociaux expliquant les limites de la réforme agraire.

\section{MOTS CLÉS}

Politiques Foncières. Histoires

Agraires. France. Brésil.

\section{ABSTRACT}

This article proposes a comparative analysis of two countries, France and Brazil, where agriculture occupies a particular place, with contrasting trajectories and challenges. The analysis focuses on the question of land ownership that has strongly influenced the history of these two countries and that, takes on particular importance because of the food and environmental crises. The data come from the work of the authors conducted in parallel and together, in addition to a comparative reading of the statistical and bibliographic sources about the history of land ownership and agricultural policies in the two countries. The first part of the study about land ownership challenges presents a trend towards the dualization of the farmers in each country based on highly contrasting routes. In Brazil, the number of small farms in situations of poverty and insecurity of land ownership is increasing more quickly than the opportunity for land ownership and the economic pie reserved for them. In France, increasingly fewer and larger farms account for most of the lands liberated in a context of strong competition for land. The review of the history of land ownership and agricultural policies, conducted in the second part of the study, shows that despite the similar objectives exhibited on both sides of the Atlantic, only France implemented a true land re-structuring in benefit of midsize family farming, which constitutes the base of agricultural modernization. In Brazil, land policy is in the first place the historic result of power relations between agrarian elites and social movements, which explains the limits of agrarian reform

\section{KEYWORDS}

Land Ownership. Agricultural

History. France. Brazil

Recebido em: 21/03/2014

Aprovado em: 06/03/2015 